\title{
The Local Structure of the Spectrum of the One-Dimensional Schrödinger Operator
}

\author{
S. A. Molčanov
}

Department of Mathematics, Moscow State University, Moscow U-234, USSR

Abstract. Let $H_{V}=-\frac{d^{2}}{d t^{2}}+q(t, \omega)$ be an one-dimensional random Schrödinger operator in $\mathscr{L}^{2}(-V, V)$ with the classical boundary conditions. The random potential $q(t, \omega)$ has a form $q(t, \omega)=F\left(x_{t}\right)$, where $x_{t}$ is a Brownian motion on the compact Riemannian manifold $K$ and $F: K \rightarrow R^{1}$ is a smooth Morse function, $\min _{K} F=0$. Let $N_{V}(\Delta)=\sum_{E_{i}(V) \in \Delta} 1$, where $\Delta \in(0, \infty), E_{i}(V)$ are the eigenvalues of $H_{V}$. The main result (Theorem 1) of this paper is the following. If $V \rightarrow \infty, E_{0}>0$, $k \in Z_{+}$and $a>0$ ( $a$ is a fixed constant) then

$$
P\left\{N_{V}\left(E_{0}-\frac{a}{2 V}, E_{0}+\frac{a}{2 V}\right)=k\right\} \underset{V \rightarrow \infty}{\longrightarrow} e^{-a n\left(E_{0}\right)}\left(\operatorname{an}\left(E_{0}\right)\right)^{k} \mid k !,
$$

where $n\left(E_{0}\right)$ is a limit state density of $H_{V}, V \rightarrow \infty$. This theorem mean that there is no repulsion between energy levels of the operator $H_{V}, V \rightarrow \infty$.

The second result (Theorem 2) describes the phenomen of the repulsion of the corresponding wave functions.

1.

In a series of latest works in physics (see [1]) the phenomenon of the repulsion of the energy levels in the spectrum of complicated (random) quantum systems was discussed. The formal definitions are the following.

Let $\mathbf{H}_{V}$ be the family of the Hamiltonians describing the behaviour of the system in the volume $V$ and let $E_{1}^{(V)}<E_{2}^{(V)} \leqq \ldots$ be the corresponding energy levels. In various interesting cases these levels are thickening in the limit and moreover for every $\alpha>0 E_{\alpha|V|}^{(V)} \rightarrow E_{\alpha}$ as $|V| \rightarrow \infty$.

We shall consider two neighbour levels $E_{n}^{(V)}$ and $E_{n+1}^{(V)}$, where $n \sim \alpha|V|$. It is natural to suppose that the normalized "spectral split" $\Delta_{n}=\left(E_{n+1}-E_{n}\right)$ / $M\left(E_{n+1}-E_{n}\right)$ has a limit distribution as $|V| \rightarrow \infty$, i.e. there exists

$$
\lim _{|V| \rightarrow \infty} \mathbf{P}\left\{\Delta_{n}<x\right\}=G(x) .
$$


If $G(x)=o(x)$ when $x \rightarrow 0$ in this case we deal with the repulsion of the levels (near $\left.E_{\alpha}\right)$; if $G(x) \sim c x$ we say that an interaction of the levels does not exist near $E_{\alpha}$; in the case $G(x) / x \underset{x \rightarrow 0}{\longrightarrow} \infty$ we may say that there is an attraction between levels (or that the levels show a tendency to group).

It is natural to study several levels near $E_{\alpha}$. Mathematically this problem is reduced to the analysis of the joint limit distribution of the several neighbour spectral splits $\Delta_{n}, \Delta_{n+1}, \ldots, \Delta_{n+k-1}$.

As far as it is known to the author no rigorous result in this field has yet been published. However in the so called Wigner Gaussian symmetrical ensemble $\mathbf{H}_{n}=\left(\xi_{i j}\right), i, j=1,2, \ldots, n\left(\xi_{i j}, i \geqq j\right.$ are an independent Gaussian random values) the limit distribution function averaged in all splits was found, i.e.

$$
\bar{G}(x)=\lim _{n \rightarrow \infty} \frac{1}{n} \sum_{k=1}^{n} \mathbf{P}\left\{\Delta_{k}<x\right\}
$$

and the existence of repulsion was established [2-4].

For the unordered structures the spectrum of which coincides with that of the Schrödinger operator with the random potential the repulsion of the levels was also asserted in [5]. A number of physics works following [5] were based on the results of [5], but it turned out that [5] was false. It is possible to prove the absence of the interaction between the levels in unordered one-dimensional structures for a large class of random stationary potentials, in particular, for $\delta$-potential explored in [5]. Moreover it is possible to analyse the local structure of the spectrum near the fixed point $E_{\alpha}$ in full. This spectrum proves to be a Poisson flow near $E_{\alpha}$ on the natural scale, i.e. the neighbour spectral splits (asymptotically as $|V| \rightarrow \infty)$ are independent and have exponential distribution.

Our paper contains the proof of the above formulated results and is close to $[6,7]$.

For the sake of convenience of the references to [7] we narrow the class of the studied potentials but our results remain true for the Kronig-Penny potential and for the potential of the "white noise" type.

\section{2.}

Now we pass on to exact formulations. We consider the Schrödinger operator of the Markov type which has been introduced in $[6,7]$, namely

$$
\mathbf{H}=-\frac{d^{2}}{d t^{2}}+F\left(x_{t}(\omega)\right), \quad t \in R^{1}, \quad \omega \in \Omega
$$

Here $\Omega$ is the probability space with the measure $\mathbf{P}$ (this space may be identified with the ensemble of all the realizations of the process $x_{t}, t \in R^{1}, x_{t}(\omega)$ is the Brownian motion on the compact Riemannian manifold $K$ and has the generating operator 4 ). The invariant measure of $x_{t}$ is the natural Riemannian measure. Taking $d x$ to be the initial distribution we turn $x_{t}$ into stationary Markov process with "good" mixing properties. The function $F: K \rightarrow R^{1}$ is smooth $\left(C^{\infty}\right)$ and "nonflat" (see $[6,7]$ ). The last is fulfilled when $F$ has a finite number of nondegenerate (Morse) critical points. 
If $\min _{x \in K} F(x)=0,\|F\|=1$, then $[6,7]$ the spectrum $S$ of the unique selfadjoint extension of $H$ on $\mathscr{L}^{2}\left(R^{1}\right)$ coincides with the half-axis $[0, \infty)$. Let us consider the restriction $H$ to $\mathscr{L}^{2}(-V, V)$.

This restriction is defined by classical boundary conditions. We analyze here the corresponding spectral problem

$$
\begin{aligned}
\mathbf{H}_{V} \psi & =-\frac{d^{2} \psi}{d t^{2}}+F\left(x_{t}\right) \psi=E \psi, \quad t \in(-V, V), \\
\psi(-V) & =\psi(V)=0 .
\end{aligned}
$$

Let $0<E_{1}(V, \omega)<E_{2}(V, \omega)<\ldots$ be the eigenvalues (the levels) of the problem (2), $\psi_{E_{i}}, i=1,2, \ldots$ are the corresponding eigenfunctions (the wave functions). The existence of the limit spectral distribution function

$$
\mathbf{N}(E)=\lim _{V \rightarrow \infty} \frac{1}{2 V} \sum_{E_{i}(V) \leqq E} 1
$$

was proved in [8] for more general situation. Moreover under our conditions there exists the continuous state density $n(E)=\frac{d \mathbf{N}(N)}{d E}$ and besides $n(E)>0$ if $E>0$ (see Proposition 1).

\section{3.}

The analysis of the phase of the equation $\mathbf{H} \psi=E \psi$ is the clue to the analysis of all the basic spectral characteristics of the operator $\mathbf{H}\left(\right.$ or $\left.\mathbf{H}_{V}\right)$. If $\theta_{E}(t)=\operatorname{arcctg} \psi^{\prime} / \psi$ then

$$
\frac{d \theta_{E}}{d t}=\cos ^{2} \theta_{E}+\left(E-F\left(x_{t}\right)\right) \sin ^{2} \theta_{E}, \quad \theta_{E} \in S^{1} .
$$

Here $S^{1}$ is one-dimensional torus, i.e. the interval $[0, \pi]$ with the identified ends. The "two-dimensional process" $\left(x_{t}, \theta_{E}(t)\right)$ is the Markov diffusion one on $K \times S^{1}$ and its infinitesimal operator is

$$
A_{E}=\frac{1}{2} \Delta+\left[\cos ^{2} \theta+(E-F(x)) \sin ^{2} \theta\right] \frac{\partial}{\partial \theta} .
$$

According to Hörmander theory (see [6,7]), the parabolic equation $\partial p / \partial t=A_{E} p$ has the fundamental solution in the cylinder $(0, \infty) \times\left(K \times S^{1}\right)$, this solution is smooth in all arguments and besides for $E>0$ (i.e. on the spectrum)

$$
p_{E}\left(t,(x, \theta),\left(x_{1}, \theta_{1}\right) \geqq \varepsilon(t, E)>0,\right.
$$

if $t \geqq t_{0}(E)$. The last expression (the strong form of the Döeblin condition) guarantees us the existence of the limit

$$
\pi_{E}(x, \theta)=\lim _{t \rightarrow \infty} p_{E}(t,(\cdot, \cdot),(x, \theta)) \geqq \varepsilon\left(E, t_{0}\right)
$$


with the exponential speed, i.e.

$$
\left|p_{E}(t,(\cdot, \cdot),(x, \theta))-\pi_{E}(x, \theta)\right| \leqq c(E) e^{-\delta(E) t 1} .
$$

The constants in (7), $\left(7^{\prime}\right)$ are strictly positive in every open interval of the axis $(0, \infty)$.

4.

Let us fix the point $E_{0} \in(0, \infty)$ and its certain neighbourhood $U_{0} \subset(0, \infty)$. All the following discussions will be conducted in $U_{0}$, the dependence of constants on $V_{0}$ will be implied.

Let us prove the existence of the state density $n(E)$ in a stronger form. Let $\Delta=(E, E+h) \subset U_{0}$

$$
N_{V}(\Delta)=N_{V}(E+h)-N_{V}(E)=\sum_{E_{i}(V) \in \Delta} 1
$$

Proposition 1. For $V \rightarrow \infty$ and every $\varepsilon>0$

$$
\begin{aligned}
M \frac{\mathbf{N}_{V}(\Delta)}{2 V} & =\int_{\Delta} d E \int_{K \times S^{1}} \pi_{E}(x, \theta) \sin ^{2} \theta \pi_{E}(x, \pi-\theta) d x d \theta+o\left(\frac{\ln ^{1+\varepsilon} V}{V}\right) \\
& =\int_{\Delta} n(E) d E+o\left(\frac{\ln ^{1+\varepsilon} V}{V}\right) .
\end{aligned}
$$

Proof. Let us use the general method of [6] and [7, Sect. 3]. We introduce "the quasi-solution" $\tilde{\psi}_{E, t}(s)$ of the equation $\mathbf{H} \psi=E \psi$ satisfying this equation for $s \neq t$ with the boundary conditions

$$
\tilde{\psi}_{E, t}(-V)=\tilde{\psi}_{E, t}(V)=0
$$

and having the unit amplitude $\tilde{r}_{E, t}=\sqrt{\tilde{\psi}^{2}+\left(\frac{d \tilde{\psi}}{d s}\right)^{2}}$ for $s=t$. This "quasi-solution" will be a wave function if we suppose

$$
\tilde{\theta}_{E, t}(t+0)=\tilde{\theta}_{E, t}(t-0) \text {. }
$$

The last formula (for any $t$ ) uniquely defines the spectrum $E_{i}(V)$. Clearly, for any $t \in(-V, V)$

$$
\begin{aligned}
M \frac{\mathbf{N}_{V}(\Delta)}{2 V} & =\frac{1}{2 V} \int_{-V}^{V} d s M \sum_{E_{i}(V) \in \Delta} \frac{\tilde{\psi}_{E_{l}, t}^{2}(s)}{\int_{-V}^{V} \tilde{\psi}_{E_{i}, t}^{2} d s} \\
= & \frac{1}{2 V} \int_{-V}^{V} M \int_{\Delta} d E \delta\left(\tilde{\theta}_{E}(t+0)-\tilde{\theta}_{E}(t-0)\right)\left|\frac{\partial}{\partial s}\left(\tilde{\theta}_{E}(t+0)-\tilde{\theta}_{E}(t-0)\right)\right| \\
& \cdot \tilde{\psi}_{E, t}^{2}(s) / \int_{-V}^{V} \tilde{\psi}_{E, t}^{2} d s
\end{aligned}
$$

1 In future we shall suppose that $c_{i}, \varepsilon_{i}, \delta_{i}$ are positive constants independent of $V$. We also suppose $V$ to be sufficiently large 
Let us choose $t=s$ in the inner integral, then $\tilde{\psi}_{s}^{2}(s)=\sin ^{2} \theta_{E}(s)$. By the general theorem of [7, Sect. 3] (see also Lemma 1, [6]) we find that

$$
\begin{aligned}
M \frac{\mathbf{N}_{V}(\Delta)}{2 V}= & \frac{1}{2 V} \int_{-V}^{V} d s \int_{\Delta} d E \int_{K \times S^{1}} p_{E}(V-s,(K, 0),(x, \theta)) \\
& \cdot \sin ^{2} \theta p_{E}(V+s,(K, 0),(x, \pi-\theta)) d x d \theta .
\end{aligned}
$$

The notation $p_{E}\left(t,\left(K, \theta_{0}\right),(\cdot, \cdot)\right)$ means the transition density of the process $\left(x_{t}, \theta_{E}(t)\right)$ with the uniform (in $\left.x\right)$ initial distribution and the initial phase $\theta_{0}$.

Using $\left(7^{\prime}\right)$ we immediately obtain (8), since $\pi_{E}(x, \theta)>0$, for $E \in U_{0}$, then $n(E)>0$ on the spectrum $\mathscr{S}=(0, \infty)$.

\section{5.}

Now we can rigorously formulate the basic result of our work.

Theorem 1. For fixed $a_{1}<b_{1} \leqq a_{2}<b_{2} \leqq \ldots \leqq a_{n}<b_{n}$ and nonnegative integers $k_{1}, \ldots, k_{n}$

$$
\begin{gathered}
\lim _{V \rightarrow \infty} \mathbf{P}\left\{\mathbf{N}_{V}\left(\Delta_{1}\right)=k_{1}, \ldots, \mathbf{N}_{V}\left(\Delta_{n}\right)=k_{n}\right\} \\
=\exp \left\{-n\left(E_{0}\right)\left[\left(l_{1}-a_{1}\right)+\ldots+\left(l_{n}-a_{n}\right)\right]\right\} \cdot \frac{\left[n\left(E_{0}\right)\left(b_{1}-a_{1}\right)\right]^{k_{1}}}{k_{1} !} \ldots \frac{\left[n\left(E_{0}\right)\left(b_{n}-a_{n}\right)\right]^{k_{n}}}{k_{n} !}
\end{gathered}
$$

where $\Delta_{i}=\left(E_{0}+\frac{a_{i}}{2 V}, E_{0}+\frac{b_{i}}{2 V}\right), i=1,2, \ldots, n$.

The expression (9) holds uniformly in $a_{i}, b_{i} ;\left|a_{i}\right|,\left|b_{i}\right| \leqq A, k_{i} \leqq M$ ( $A, M$ are fixed). In other words the random point process $\mathbf{N}_{V}^{*}(E)=\mathbf{N}_{V}\left(E_{0}, E_{0}+\frac{E}{2 V}\right)$ is an asymptotically Poisson one (with the parameter $n\left(E_{0}\right)$ ) in the sense of the convergence of the finite-dimensional distributions.

We shall prove it along the following lines. Let us consider another problem $\left(2^{\prime}\right)$ which consists of $k$ problems, where $k=k(V)=\left[\ln ^{1+\varepsilon} V\right]$. We consider the points

$$
t_{0}=-V, \quad t_{1}=-V+\frac{2 V}{k}, \ldots, t_{i}=-V+\frac{2 V_{i}}{k}, \ldots, t_{k}=V
$$

Let us construct the neighbourhoods of radius $\ln ^{1+\varepsilon} V$ around every point $t_{1}, t_{2}, \ldots, t_{k-1}$. In other words, we have introduced the intervals

$$
\mathscr{D}_{i}=\left(\lambda_{i}, \mu_{i}\right)=\left(t_{i}-\ln ^{1+\varepsilon} V, t_{i}+\ln ^{1+\varepsilon} V\right), \quad i=1,2, \ldots, k-1, \quad \mu_{0}-V, \quad \lambda_{k}=V .
$$

The set $[-V, V] \backslash \bigcup_{i=1}^{k} \mathscr{D}_{i}$ is divided into $k$ intervals

$$
\left(\mu_{0}, \lambda_{1}\right)=I_{1}, \quad\left(\mu_{1}, \lambda_{2}\right)=I_{2}, \ldots,\left(\mu_{k-1}, \lambda_{k}\right)=I_{k}
$$

and the problem $\left(2^{\prime}\right)$ is: in every interval $I_{1}, I_{2}, \ldots, I_{k}$ it is necessary to find the functions $\psi$ such that

$$
\mathbf{H}^{(j)} \psi=E \psi, \quad s \in I_{j}, \quad \psi\left(\mu_{j-1}\right)=\psi\left(\lambda_{j}\right)=0 ; \quad j=1,2, \ldots, k .
$$


We shall denote the corresponding eigenvalues (levels) and the wave functions by $E_{i}^{(j)}$ and $\psi_{i}^{(j)}$ resp. Let

$$
\overline{\mathbf{N}}_{V}(\Delta)=\sum_{j} \sum_{E_{i}^{(j)} \in \Delta} 1
$$

First we shall establish, that

$$
\mathbf{P}\left\{\overline{\mathbf{N}}_{V}(\Delta)=\mathbf{N}_{V}(\Delta)\right\} \underset{V \rightarrow \infty}{\longrightarrow} 1, \quad \Delta=\left(E_{0}+\frac{a}{2 V}, E_{0}+\frac{l}{2 V}\right),
$$

using the fact of the exponential decreasing of the eigenfunctions on $[-V, V]$ (one of the results of [7]). This expression means, that the spectra of the problems (2) and $\left(2^{\prime}\right)$ "almost coincides" in the neighbourhood of the radius $O\left(\frac{1}{V}\right)$. Since

$$
\overline{\mathbf{N}}_{V}(\Delta)=\sum_{j=1}^{k} \sum_{E_{i}^{(j)} \in \Delta} 1=\sum_{j=1}^{k} \mathbf{N}_{V}^{(j)}(\Delta)
$$

and the terms in the last sum are equally distributed, "almost independent" and "infinitely small", we came to the standard scheme. Unfortunately, we cannot obtain the Poisson limit distribution for $\mathbf{N}_{V}(\Delta)$ from this scheme. A priori we can only achieve the complex Poisson distribution. The final and most complicated step is connected with the analysis of the second moment $M \mathbf{N}_{V}^{2}(\Delta)$.

6.

At the first stage the two following propositions will be of major importance.

Proposition 2. (Essentially coincides with Theorem 4, Sect. 6 [7].) For fixed $\varepsilon>0$ there exists $\delta>0$ such that the probability of $\bar{A}_{V}^{\varepsilon, \delta}$ tends to zero as $V \rightarrow \infty$, where $A_{V}^{\varepsilon, \delta}$ $=\left\{\right.$ To every normalized wave function $\psi_{E_{i}}(s), E_{i} \in U_{0}, s \in[-V, V]$ corresponds the point $\tau\left(\psi_{E_{i}}\right)$, "the center of the support" of $\psi_{E_{i}}(\cdot)$, so that for all $s$ : $\left|s-\tau\left(\psi_{E_{2}}\right)\right| \geqq \ln ^{1+\varepsilon} V$ we have the estimate

$$
\left.r\left(\psi_{E_{i}}\right)=\sqrt{\psi_{E_{i}}^{2}+\left(\psi_{E_{i}}^{\prime}\right)^{2}} \leqq \exp \{-\delta|s-\tau|\} .\right\} .
$$

In fact the stronger result was proved in [7], namely, that $\sum_{n} \mathbf{P}\left(\bar{A}_{n}^{\varepsilon, \delta}\right)<\infty$. One should note that the point $\tau\left(\psi_{E_{i}}\right)$ must be chosen measurably $(\Omega, \mathbf{P})$ (which was not emphasized in [7]). For example, we may put $\tau\left(\psi_{E_{i}}\right)=$ the first rational point in $[-V, V]$ (the numeration is standard) such that $\left|\psi_{E_{i}}\right| \geqq \frac{1}{\sqrt{2 V}}$.

Proposition 3. The number of the levels of the problem (2) getting in $\Delta=(E-h, E+h)$ coincides with the maximum dimension of the linear manifold $\mathscr{L}^{\Delta}$ of the smooth functions $\psi$ (with the null boundary conditions) such that

$$
\begin{aligned}
\left(\left(\mathbf{H}^{V}-E\right) \psi, \psi\right) & =\int_{-V}^{V}\left[\left(\psi^{\prime}\right)^{2}+\psi^{2}\left(F\left(x_{s}\right)-E\right)\right] d s \\
& \leqq h^{2}(\psi, \psi)=h^{2} \int_{-V}^{V} \psi^{2}(s) d s .
\end{aligned}
$$


This lemma of the abstract theory of the linear operators belongs to Glasman (see [9, Chap. 1, Theorem $\left.3^{\text {bis }}\right]$ ). Proposition 3 is also true in case of the problem $\left(2^{\prime}\right)$, one should accordingly modify the boundary conditions.

Let us return to Theorem 1. For the sake of shortness we limit ourselves to the one interval $\Delta=\left(E_{0}-\frac{a}{2 V}, E_{0}+\frac{a}{2 V}\right)$. Technical details necessary for the general case will be described below (Propositions 5', 6, Theorem 1).

Let us consider the intervals $\tilde{\mathscr{D}}_{i}=\left(t_{i}-2 \ln _{V}^{1+\varepsilon}, t_{i}+2 \ln _{V}^{1+\varepsilon}\right), i=1,2, \ldots, k-1$ which are twice as large as $\mathscr{D}_{i}, i=1,2, \ldots, k-1$ introduced in Sect. 5 .

Proposition 4. If $\Delta=\left(E_{0}-a / 2 V, E_{0}+a / 2 V\right)$, then

$$
M \sum_{E_{i} \in \Delta} \int_{\substack { k-1 \\
\begin{subarray}{c}{i=1 \\
i=1{ k - 1 \\
\begin{subarray} { c } { i = 1 \\
i = 1 } }\end{subarray}} r_{E_{i}}^{2}(s) d s \leqq C(a, \varepsilon) \frac{\ln ^{2+2 \varepsilon} V}{V}\left(1+O\left(\frac{1}{V}\right)\right) .
$$

Here $r_{E_{i}}$ are the amplitudes of the wavefunctions $\psi_{E_{i}}$ of the problem (2).

The proof repeats the discussion in Proposition 1 almost literally. Namely

$$
\begin{aligned}
& M \sum_{E_{i} \in \Delta} \int_{\substack{\bigcup_{i}-1 \\
\bigcup_{1} \tilde{\mathscr{D}}_{i}}} d s \int_{\Delta} d E \int_{K \times S^{1}} p_{E}(V-s,(K, 0)(x, \theta)) \\
& \cdot p_{E}(V+s,(K, 0),(x, \pi-\theta)) d x d \theta \\
&= \int_{\cup \tilde{\mathscr{D}}_{2}} d s \int_{\Delta} d E \int_{K \times S^{1}}\left[\pi_{E}(x, \theta) \pi_{E}(x, \pi-\theta)+o\left(e^{-V^{1-\varepsilon_{1}}}\right)\right] d x d \theta \\
&= k \ln ^{1+\varepsilon} V \frac{2 a}{V} n\left(E_{0}\right)+o\left(e^{-\sqrt{V}}\right) \\
& \leqq \ln ^{2+2 \varepsilon} V \\
& V
\end{aligned}
$$

We have used both the smoothness $\pi_{E}$ in $E$ and the fact, that

$$
\left.\min \left(V-t, t \in \cup \tilde{\mathscr{D}}_{i}\right) \geqq \frac{V}{\ln ^{1+\varepsilon} V}-2 \ln ^{1+\varepsilon} V>V^{1-\varepsilon_{1}}>\sqrt{V}\right] .
$$

One should also remember that $k=k(V)=\left[\ln ^{1+\varepsilon} V\right]$.

7.

Let us compare Proposition 2 with Proposition 4.

If at least one of the points $\tau\left(\psi_{E_{i}}\right) ; E_{i} \in \Delta$ belongs to $\bigcup_{i=1}^{k-1} \mathscr{D}_{i}$ and $A_{V}^{\varepsilon, \delta}$ has occurred, then

$$
\int_{\substack{\bigcup_{i=1} \bigcup_{i=1}^{1} \tilde{\mathscr{D}}_{\imath} \\ E_{\imath} \in \Delta}} \sum_{E_{\imath}}^{2} d s \geqq 1-\int_{|s|>\ln ^{1+\varepsilon} V} e^{-\delta|s|} d s \geqq 1-C_{1} e^{-\delta \ln ^{1+\varepsilon} V} .
$$


Since $P\left\{A_{V}^{\varepsilon, \delta}\right\} \rightarrow 1$ and the mean of the left hand side random value equals to $O\left(\frac{\ln ^{2+2 \varepsilon} V}{V}\right)$ (according to Proposition 4) then

i.e.

$$
\mathbf{P}\left\{\text { the points } \mathrm{T}_{i}=\tau\left(\psi_{E_{i}}\right) \in \bigcup_{i=1}^{k=1} \mathscr{D}_{i}\right\} \underset{V \rightarrow \infty}{\longrightarrow} 0,
$$

$$
\mathbf{P}\left\{\tau_{i} \text { do not get in } \bigcup_{i=1}^{k-1} \mathscr{D}_{i}\right\} \underset{V \rightarrow \infty}{\longrightarrow} 1 .
$$

Moreover, the same discussions show that no one of the points $\tau_{i}=\tau\left(\psi_{E_{2}}\right), E_{i} \in \Delta$ will be at the distance $\leqq \ln ^{1+\varepsilon} V$ from $\bigcup_{i=1}^{k-1} \mathscr{D}_{i}$ with the probability $q_{V}: q_{V} \underset{V \rightarrow \infty}{\longrightarrow} 1$. In other words it is asymptotically reliable that all $\psi_{E_{i}}, E_{i} \in \Delta$ admit the estimate $r_{E_{l}} \leqq e^{-\delta \ln ^{1+\varepsilon} V}$ on $\bigcup_{i=1}^{k-1} \mathscr{D}_{i}$. We shall denote this event by $B_{V}^{\varepsilon, \delta}$.

Let us consider the smooth cutting function $g_{V}(s)$ equalling to 0 on $\bigcup_{i=1}^{k-1} \mathscr{D}_{i}$ and to 1 outside the 1 -neighbourhood of $\bigcup_{i=1}^{k-1} \mathscr{D}_{i}$. The functions $\bar{\psi}_{E_{i}}(s)=g_{V}(s) \psi_{E_{t}}(s), E_{i} \in \Delta$ satisfy the null conditions at the ends of the intervals $\mathscr{D}_{i}$, i.e. at the points $\mu_{i}, \lambda_{i}$, $i=1,2, \ldots, k-1$. Let us pull the finite-dimensional subspace $\tilde{\mathscr{L}}^{\Delta}$ on these functions. If the above described event $B_{V}^{\varepsilon, \delta}$ occurs then

and

$$
\left(\sum c_{i} \bar{\psi}_{E_{i}}, \sum c_{i} \bar{\psi}_{E_{i}}\right)=\sum_{i=1}^{N_{V}(\Delta)} c_{i}^{2}+R_{1}(V)
$$

$$
\left(\left(\mathbf{H}-E_{0}\right) \sum c_{i} \bar{\psi}_{E_{i}}, \sum c_{i} \bar{\psi}_{E_{i}}\right)<\left(\frac{a}{2 V}\right)^{2} \sum_{i=1}^{\mathbf{N}_{V}(\Delta)} c_{i}^{2}+R_{2}(V) .
$$

The remainders $R_{1}(V)$ and $R_{2}(V)$ are estimated by the sums of the integrals from $r_{E_{i}}^{2}$ over $\bigcup_{i=1}^{k-1} \overline{\mathscr{D}}_{i}$, where $\overline{\mathscr{D}}_{i}$ are the $I$-neighbourhoods of $\mathscr{D}_{i}, i=1,2, \ldots, k-1$. Therefore $\left|R_{1,2}(V)\right| \leqq C e^{-\delta \ln ^{1+\varepsilon}} V \cdot \mathbf{N}_{V}(\Delta)$.

Using the Čebyšev's inequality and putting $\sum_{i=1}^{\mathbf{N}_{V}(\Delta)} c_{i}^{2}=1$ without loss of generality we achieve that $\operatorname{dim} \tilde{\mathscr{L}}^{\Delta} \geqq \mathbf{N}_{V}(\Delta)$ with the probability tending to 1 as $V \rightarrow \infty$. In other words (see Proposition 3) it is asymptotically reliable that $\overline{\mathbf{N}}_{V}(\Delta) \geqq \mathbf{N}_{V}(\Delta)$. But as it is easy to see the inequality $\mathbf{N}_{V}(\Delta) \leqq \mathbf{N}_{V}(\Delta)$ always holds true, hence it is asymptotically reliable that $\overline{\mathbf{N}}_{V}(\Delta)=\mathbf{N}_{V}(\Delta)$ or which is the same $\overline{\mathbf{N}}_{V}(\Delta)$ $-\mathbf{N}_{V}(\Delta) \underset{V \rightarrow \infty}{\stackrel{\mathbf{P}}{\longrightarrow}} 0$. In terms of the characteristic functions the last expression means that

$$
M e^{i \lambda \mathbf{N}_{V}(\Delta)}-M e^{i \lambda{ }_{j} \sum_{j=1}^{k} \mathbf{N}_{V}^{(j)}(\Delta)} \underset{V \rightarrow \infty}{\longrightarrow} 0
$$

8.

The values $\mathbf{N}_{V}^{(j)}(\Delta)$ are measurable with respect to $\sigma$-algebras generated by the process $x_{t}$ (i.e. by the potential) on the intervals $I_{1}=\left(\mu_{0}, \lambda_{1}\right), I_{2}=\left(\mu_{1}, \lambda_{2}\right), \ldots$, $I_{k}=\left(\mu_{k-1}, \lambda_{k}\right)$. Since the distance between these intervals is not less than $2 \ln ^{1+\varepsilon} V$ 
and $x_{t}$ satisfies the exponential mixing condition then the general facts of the theory of the sums of the weakly dependent random variables (see [10], 19.17 and 18.4.2) yields that

$$
\left|M e^{i \lambda \sum_{J=1}^{k} \mathbf{N}_{V}^{(J)}(\Delta)}-\prod_{j=1}^{k} M e^{i \lambda \mathbf{N}_{V}^{(j)}(\Delta)}\right| \leqq 4 k C e^{-\delta_{1} \ln ^{1+\varepsilon} V} \leqq C_{1} e^{-\frac{\delta^{1}}{2} \ln { }^{1+\varepsilon} V}
$$

But the values $\mathbf{N}_{V}^{(j)}(\Delta)$ are equally distributed [except the extreme terms $\mathbf{N}_{V}^{(1)}(\Delta)$ and $\left.\mathbf{N}_{V}^{(k)}(\Delta)\right]$ and besides

$$
M \mathbf{N}_{V}^{(j)}(\Delta)=\frac{2 a n\left(E_{0}\right)}{k(V)}+o\left(\frac{\ln V}{V}\right), \quad j=2, \ldots, k-1
$$

(Proposition 1).

According to (14) we can forget the interdependence of $\mathbf{N}_{V}^{(j)}$ and consider them independent and equally distributed [the extreme terms $\mathbf{N}_{V}^{(j)}(\Delta)$ and $\mathbf{N}_{V}^{(k)}(\Delta)$ give the asymptotically negligible contributions since their means vanish as $V \rightarrow \infty$, $k(V) \rightarrow \infty]$.

Let us now use the following simple limit theorem.

Proposition 5. Let us have a sequence of the series of independent integer-valued and equally distributed in each series random values $\xi_{n 1}, \xi_{n 2}, \ldots, \xi_{n n}, n=1,2, \ldots$ with $M \xi_{n i}=\frac{\lambda}{n}, i=1,2, \ldots, n$. Then for some subsequence $\left\{n_{j}\right\}$ for all $k \geqq 1$ there exists $\lim _{n \rightarrow \infty} n \mathbf{P}\left\{\xi_{n i}=k\right\}=p_{k}$ and as $n \rightarrow \infty$ the distribution of $S_{n}=\sum_{i=1}^{n} \xi_{n i}$ converges to the limit distribution with the characteristic function

$$
\varphi(\lambda)=\lim _{n_{i} \rightarrow \infty} M e^{i \lambda S_{n}}=e^{\sum_{k=1}^{\infty} p_{k}\left(e^{i k \lambda}-1\right)},
$$

where $\varphi^{\prime}(\lambda)_{\mid \lambda=0}=i \lambda=i \sum_{k=1}^{\infty} k p_{k}$. The limit distribution is the Poisson one with the parameter $\lambda$ if $p_{1}=\lambda, p_{2}=p_{3}=\ldots=0$.

Being standard the proof is omitted.

Let us note that in general case the formula (15) defines a complex Poisson distribution. The vector version of Proposition 5 is also true.

Proposition $5^{\prime}$. If $\xi_{n 1}, \xi_{n 2}, \ldots, \xi_{n n}, n=1,2, \ldots$ are a sequence of the series of the vectors which are independent in each series and take the values in $Z_{+}^{v}, M \xi_{n i}=\frac{a}{n}$, $a \in R_{+}^{v}$, then (it is possible, for some subsequence) there are $\lim _{n \rightarrow \infty} n P\left\{\xi_{n i}=k\right\}=p_{k}$, $k \in Z_{+}^{v}$ and

$$
\lim _{n \rightarrow \infty} M e^{i\left(\lambda, S_{n}\right)}=\exp \left\{\sum_{k \in Z_{+}^{v}}\left(e^{i(\lambda, k)}-1\right)\right\} .
$$


9.

Using the formulae (13), (14) and Proposition $5\left(5^{\prime}\right)$ we immediately come to the following "half-finished" variant of Theorem 1.

Theorem 1'. For fixed $a_{1}<b_{1} \leqq a_{2}<b_{2} \leqq \ldots \leqq a_{n}<b_{n}$ and

$$
\begin{aligned}
\Delta_{i} & =\left(E_{0}+\frac{a_{i}}{2 V}, E_{0}+\frac{b_{i}}{2 V}\right), \quad i=1,2, \ldots, n \\
\lim _{V \rightarrow \infty} M e^{\sum_{k=1}^{n} i \lambda_{k} \mathbf{N}_{V}\left(\Delta_{k}\right)} & =e^{\sum_{m \in \mathbf{Z}_{+}^{n}} p m\left(e^{l(\lambda, m)}-1\right)} .
\end{aligned}
$$

The limit in (16) exists a priori only for some subsequence $\left\{V_{i}\right\}, V_{i} \rightarrow \infty$, and the constants $p_{m}, m \in Z_{+}^{n}$ depend both on $\left\{V_{i}\right\}$ and $\left(a_{i}, b_{i}\right), i=1,2, \ldots, n$.

Theorem $1^{\prime}$ roughly speaking states that the point flow $E_{i}(V)$ is a complex Poisson near $E_{0}$ on the scale $\frac{1}{2 V}$.

Since the spectrum $E_{i}(V)$ is simple, we think it likely that the word "complex" may be removed. But we cannot exclude the possibility of the existence of the groups of $E_{i}(V)$ the distance between which has the order $o\left(\frac{1}{V}\right)$ and therefore they will be "glued" by our normalization as $V \rightarrow \infty$. We need some additional information on senior moments of $\mathbf{N}_{V}(\Delta)$. The following proposition is obvious.

Proposition 6. We keep the notations of Proposition 5. Let $\xi_{n i}=\left(\xi_{n i}^{(1)}, \ldots, \xi_{n i}^{(v)}\right)$ and

$$
\begin{gathered}
M \xi_{n i}=\left(\frac{\lambda_{1}}{n}, \ldots, \frac{\lambda_{v}}{n}\right), \quad M\left[\xi_{n i}^{(j)}\right]^{2}=\frac{\lambda_{j}}{n}+o\left(\frac{1}{n}\right), \\
M \xi_{n i}^{(j)} \xi_{n i}^{(l)}=o\left(\frac{1}{n}\right)
\end{gathered}
$$

for $j \neq l, j, l=1,2, \ldots, v$. Then

$$
M e^{i\left(t, S_{n}\right)} \underset{n \rightarrow \infty}{\longrightarrow} e^{\sum_{j=1}^{v} \lambda_{J}\left(e^{i t_{j}}-1\right)}
$$

i.e. the components of the vector $S_{n}$ are asymptotically independent Poisson random values with the parameters $\lambda_{j}, j=1,2, \ldots, v$.

10.

In order to finish the proof of Theorem 1 we are left to verify (by the previous assertion) that $M \mathbf{N}_{V}^{2}(\Delta)=M \mathbf{N}_{V}(\Delta)+o\left(\frac{1}{V}\right)$ and for $j \neq l$

$$
M \mathbf{N}_{V}\left(\Delta_{j}\right) \mathbf{N}_{V}\left(\Delta_{l}\right)=o\left(\frac{1}{V}\right) .
$$

We shall study $M \mathrm{~N}_{V}^{2}(\Delta)$ at great length, the covariance is analyzed similarly and even simpler. This problem is technically close to [7, Sect. 5], where the 
variance of the spectral measure of $\mathbf{H}^{V}$ was studied. In both cases we may reduce our problem to that of obtaining "good" estimates of the transition density $p_{E, E^{\prime}}\left(t,\left(x, \theta_{1}, \theta_{2}\right),\left(\tilde{x}, \tilde{\theta}_{1}, \tilde{\theta}_{2}\right)\right)$ of the Markov process $\left(x_{t}, \theta_{E^{\prime}}(t), \theta_{E^{\prime}}(t)\right)$ for the large $t$ and $\left|E^{\prime}-E\right| \ll 1$.

Let us note that this process degenerates as $E^{\prime}=E$ and it is this fact that creats the main difficulty.

Let us begin as in Proposition 1

$$
\begin{aligned}
M \mathbf{N}_{V}^{2}(\Delta)= & M\left(\sum_{E_{i} \in \Delta} \int_{-V}^{V} \frac{\tilde{\psi}_{t, E_{i}}^{2}(s) d s}{\int_{-V}^{V} \tilde{\psi}_{t, E_{i}}^{2}(u) d u}\right)^{2} \\
= & M \iint_{-V}^{V} d T_{1} d T_{2} \sum_{E_{i}, E_{j} \in \Delta} \frac{\tilde{\psi}_{t, E_{i}}^{2}\left(T_{1}\right) \tilde{\psi}_{t, E_{j}}^{2}\left(T_{2}\right)}{\left(\int_{-V}^{V} \tilde{\psi}_{t, E_{i}}^{2} d s\right)\left(\int_{-V}^{V} \tilde{\psi}_{t, E_{j}}^{2} d u\right)} \\
= & M \int_{-V}^{V} d T_{1} d T_{2} \sum_{E_{i} \in \Delta} \frac{\tilde{\psi}_{t, E_{i}}^{2}\left(T_{1}\right) \tilde{\psi}_{t, E_{i}}^{2}\left(T_{2}\right)}{\left(\int_{-V}^{V} \tilde{\psi}_{t, E_{i}}^{2} d s\right)^{2}} \\
+ & \sum_{\substack{E_{i} \neq E_{j} \\
\left(E_{i}, E_{j} \in \Delta\right)}} \frac{\tilde{r}_{E_{i}}^{2} \sin ^{2} \theta_{E_{i}}\left(T_{1}\right) \tilde{r}_{E_{j}}^{2} \sin ^{2} \theta_{E_{j}}\left(T_{2}\right)}{\left(\int_{-V}^{V} \tilde{\psi}_{t, E_{i}}^{2} d s\right)\left(\int_{-V}^{V} \tilde{\psi}_{t, E_{j}}^{2} d u\right)} \\
= & M \mathbf{N}_{V}(\Delta)+M \iint_{-V}^{V} d T_{1} d T_{2} \sum_{\substack{E_{i} \neq E_{j} \\
\left(E_{i}, E_{j} \in \Delta\right)}} \ldots=M \mathbf{N}_{V}(\Delta)+R(\Delta) .
\end{aligned}
$$

The remaining term $R(\Delta)$ is not greater than

$$
\frac{1}{2} \iint_{-V}^{V} d T_{1} d T_{2} \sum_{E_{i} \neq E_{j}} \frac{\tilde{r}_{t, E_{i}}^{4}\left(T_{1}\right)+\tilde{r}_{t, E j}^{4}\left(T_{2}\right)}{\left(\int_{-V}^{V} \tilde{\psi}_{t, E_{i}}^{2} d S\right)\left(\int_{-V}^{V} \tilde{\psi}_{t, E_{j}}^{2} d u\right)} .
$$

We may divide the last integral into two ones, then choose $t=T_{1}$, i.e. $\tilde{r}_{T_{1}, E_{i}}\left(T_{1}\right)=1$ in the first one and $t=T_{2}$, i.e. $\tilde{r}_{T_{2}, E_{j}}\left(T_{2}\right)=1$ in the second one. According to the general theory of [7, Sect. 3] (see also [7, Sect. 5]) we finally obtain, that

$$
\begin{aligned}
R(\Delta) \leqq & V \int_{-V}^{V} d T \iint_{\Delta} d E d E^{\prime} \iiint_{K \times S^{1} \times \mathbf{S}^{1}} d x d \theta_{1} d \theta_{2} \\
& \cdot p_{E, E^{\prime}}\left(V-T,(K, 0,0),\left(x, \theta_{1}, \theta_{2}\right)\right) p_{E, E^{\prime}}\left(V+T,(K, 0,0),\left(x, \pi-\theta_{1}, \pi-\theta_{2}\right)\right) \\
= & 2 V \int_{-V}^{V} d T \iint_{E^{\prime}>E} d E d E^{\prime} \iiint_{K \times S^{1} \times S^{1}} d x d \theta_{1} d \theta_{2} p_{E, E^{\prime}}\left(V-T,(\cdot, \cdot, \cdot),\left(x, \theta_{1}, \theta_{2}\right)\right) \\
& \cdot p_{E, E^{\prime}}\left(V+T,(\cdot, \cdot, \cdot),\left(x, \pi-\theta_{1}, \pi-\theta_{2}\right)\right) .
\end{aligned}
$$

Let us denote the inner triple integral over $K \times S^{1} \times S^{1}$ by $\mathfrak{I}_{T}\left(E, E^{\prime}\right)$. This integral has been analyzed in [7]. It was established there that $\mathfrak{I}_{T}\left(E, E^{\prime}\right) \leqq \frac{C}{\left|E^{\prime}-E\right|^{\theta}}$, 
$\theta<1$, uniformly in $T$. This yields the following inequality [we must remember, that $\left.|\Delta|=O\left(\frac{1}{V}\right)\right]$

$$
R(\Delta) \leqq 2 V \int_{-V}^{V} d T \iint_{\Delta} d E d E^{\prime} \frac{C}{\left|E^{\prime}-E\right|^{\theta}} \leqq C_{1} V^{\theta}, \quad 0<\theta<1,
$$

and it is obvious, that the estimate of $\mathfrak{I}_{T}\left(E, E^{\prime}\right)$ being quite sufficient in [7] is insufficient for our aim. As a matter of fact, it was proved there that each small interval $\left[\right.$ of order $\left.o\left(\frac{1}{V}\right)\right]$ of axis $E$ with great probability contains not more than one "massive" atom of the spectral measure. So the mass of this atom is mush more than the summary mass of all remaining atoms in our interval. In our paper we have to prove that this interval with great probability contains not more than one atom.

\section{1.}

Regarding the above said the estimate of $\mathfrak{I}_{T}\left(E, E^{\prime}\right)$ should be defined more precisely.

Proposition 7. There are $C_{i}>0, i=1,2,3$ and $\varepsilon>0$ such that uniformly in $T: V+T$ $\geqq C_{1} \ln V, V-T \geqq C_{1} \ln V$

$$
\mathfrak{I}_{T}\left(E, E^{\prime}\right) \leqq C_{2}\left|E^{\prime}-E\right|^{\varepsilon},
$$

if $V+T<C_{1} \ln V, V-T<C_{1} \ln V$, then

$$
\mathfrak{J}_{T}\left(E, E^{\prime}\right) \leqq C_{3}\left|E^{\prime}-E\right|^{-\varepsilon} .
$$

Plainly, from (18) and (18') it follows immediately, that

$$
\begin{aligned}
R_{\Delta} & \leqq 2 V \int_{-V}^{V} d T \int_{\Delta \times \Delta} \mathfrak{I}_{T}\left(E, E^{\prime}\right) d E d E^{\prime} \\
& =2 V \int_{-V+C_{1} \ln V}^{V-C_{1} \ln V} \ldots+4 V \int_{V-C_{1} \ln V}^{V} \ldots \\
& =o\left(V^{-\varepsilon / 2}\right) ; \quad V \rightarrow \infty .
\end{aligned}
$$

The term $M \mathbf{N}_{V}\left(\Delta_{1}\right) \mathbf{N}_{V}\left(\Delta_{2}\right), \Delta_{1} \Delta_{2}=\emptyset$ is estimated just in the same way. Since $E^{\prime} \neq E$ then the term of the kind $M \mathbf{N}_{V}\left(\Delta_{i}\right)$ does not appear when computating a mixed moment therefore,

$$
M \mathbf{N}_{V}\left(\Delta_{1}\right) \mathbf{N}_{V}\left(\Delta_{2}\right) \leqq 2 V \int_{-V}^{V} d T \int_{\Delta_{1} \times \Delta_{2}} \mathfrak{J}_{T} d E d E^{\prime}=o\left(V^{-\varepsilon / 2}\right) .
$$

Both the estimates (19), (19') and Proposition 6 yield Theorem 1.

Proof of Proposition 7. Let us enumerate the results on the process $\left(x_{t}, \theta_{E^{\prime}}(t), \theta_{E}(t)\right)$. These results either were established in [7] or define the results of [7] more precisely. 
Let us put $h=E^{\prime}-E>0$.

$\alpha)$

$$
\begin{gathered}
M_{(K, \theta, \theta)} /\left.\sin \left(\left(\theta_{E+h}-\theta_{E}\right)(t)\right)\right|^{1-\varepsilon} \\
\leqq C(\varepsilon) h^{1-\varepsilon_{1}}, \quad t>0,
\end{gathered}
$$

(see [7, Corollary 2, $\left.2^{\prime}\right]$ ), $\varepsilon_{1}=\varepsilon_{1}(\varepsilon) \rightarrow 0$ for $\varepsilon \rightarrow 0$;

$\beta)$

$$
p_{E+h, E}\left(t,\left(x, \theta_{1}, \theta_{2}\right),\left(x^{\prime}, \theta_{1}^{\prime}, \theta_{2}^{\prime}\right)\right) \leqq \frac{C\left(t_{0}\right)}{h}, \quad t \geqq t_{0}>0
$$

(see [7, Proposition 8]);

$\left.\beta^{\prime}\right)$ there exists $t_{1}>0$ such that

$$
\begin{gathered}
p_{E+h, E}\left(t,\left(x, \theta_{1}, \theta_{2}\right),\left(x^{\prime}, \theta_{1}^{\prime}, \theta_{2}^{\prime}\right)\right) \\
\leqq \frac{C_{1}\left(t_{1}\right)}{\max \left(h,\left|\sin \left(\theta_{1}-\theta_{2}\right)\right|\right)}
\end{gathered}
$$

for $E>2, t \geqq t_{1}$;

$\gamma)$

$$
\mathbf{P}_{E+h, E}\left\{\sin \left(2\left(\theta_{E+h}-\theta_{E}\right)(t)\right)<0\right\} \leqq C_{2}\left(\varepsilon_{2}\right) h^{1-\varepsilon_{2}},
$$

if

$$
\begin{gathered}
\theta_{E+h}(0)=\theta_{E}(0)(\bmod \pi), \quad t>0 ; \\
\mathbf{P}_{E+h, E}\left\{\left(\theta_{E+h}(t)-\theta_{E}(t)\right) \bmod \pi \notin(0, \delta)\right\} \\
\leqq C_{3}\left(\delta, \varepsilon_{3}\right) h^{1-\varepsilon_{2}}, \quad t>0, \quad \theta_{E+h}(0)=\theta_{E}(0) .
\end{gathered}
$$

The estimate $\left.\beta^{\prime}\right)$ is stronger than $\beta$ ). The expression (21) is true for $\left(\theta_{1}-\theta_{2}\right)=O(h)$. The inequalities $\gamma$ ) and $\gamma^{\prime}$ ) were established in a less strong form in [7]. It was asserted there, that the left hand side term in (23) has an estimate $O\left(h^{\kappa}\right), \kappa>0$. In fact it was proved, that $\kappa=1 / 4-\varepsilon, \varepsilon>0$.

Let us prove $\beta^{\prime}$ ) and $\gamma^{\prime}$ ). We shall rely on the ideas of [7]. We consider the normalized process

$$
X_{t, h}=\left(x_{t}, \theta_{E}(t), w_{h}(t)=\frac{\sin \left(\theta_{E+h}-\theta_{E}\right)(t)}{h}\right) .
$$

It is easy to see that

$$
\frac{d w_{h}}{d t}=-w \frac{\sin 2 \theta_{E+h}+\sin 2 \theta_{E}}{2}(1+F-E)(t)+\cos \left(\theta_{E+h}-\theta_{E}\right)(t) \sin ^{2} \theta_{E+h}(t) .
$$

The solution of the last equation has a form

$$
\begin{aligned}
w_{h}(t)= & w_{h}(0) \exp \left\{-\int_{0}^{t} \frac{\sin 2 \theta_{E+h}+\sin 2 \theta_{E}}{2}(1+F-E) d s\right\} \\
& +\int_{0}^{t} d u \cos \left(\theta_{E+h}-\theta_{E}\right) \sin ^{2} \theta_{E+h} \exp \left\{-\int_{u}^{t} \ldots\right\} \\
= & w_{h}(0) \xi_{1}(t)+\xi_{2}(t) .
\end{aligned}
$$

Let us note that $\xi_{1}(0)=1, \xi_{2}(0)=0$ and $\left|\xi_{1}\left(t_{0}\right)\right|,\left|\xi_{2}\left(t_{0}\right)\right| \leqq C\left(t_{0}\right)$. 
We suppose that $\left|\left(\theta_{E+h}-\theta_{E}\right)(0)\right| \leqq \frac{\pi}{2}-\delta$. Then

$$
\exists\left(t_{1}>0\right):\left|\left(\theta_{E+h}-\theta_{E}\right)(s)\right| \leqq \frac{\pi}{2}-\frac{\delta}{2}, \quad s \leqq t_{1}
$$

whence

$$
\cos \left(\theta_{E+h}-\theta_{E}\right)(s) \geqq \delta_{1}>0, \quad s \in\left[0, t_{1}\right] .
$$

The values $\left(\xi_{1}\left(t_{1}\right), \xi_{2}\left(t_{1}\right), \theta_{E}\left(t_{1}\right), x_{t_{1}}\right)$ have a bounded joint destribution density $q\left(\xi_{1}^{\prime}, \xi_{2}^{\prime}, \theta^{\prime}, x^{\prime}\right)$ (this assertion should be proved with the help of the Hörmander's technique, see [7, Proposition 7]). Therefore, the joint distribution density of $\left(x_{t_{1}}, \theta_{E}\left(t_{1}\right), w_{h}\left(t_{1}\right)\right)$ equals

$$
\begin{aligned}
& \frac{1}{w_{h}(0)} \int_{0}^{\infty} q\left(\frac{\xi_{1}^{\prime}-\xi_{2}^{\prime}}{W_{h}(0)}, \xi_{2}^{\prime}, \theta^{\prime}, x^{\prime}\right) d \xi_{2}^{\prime} \\
& \leqq \frac{C\left(t_{1}\right)\|q\|}{W_{h}(0)} \leqq \frac{C_{1}\left(t_{1}\right) h}{\left|\sin \left(\theta_{E+h}-\theta_{E}\right)(0)\right|} .
\end{aligned}
$$

This inequality yields the estimate $\beta^{\prime \prime}$ ):

$$
\begin{aligned}
p\left(t_{1},\left(x, \theta_{1}, \theta_{2}\right),\left(x^{\prime}, \theta_{1}^{\prime}, \theta_{2}^{\prime}\right)\right) & \leqq \frac{C_{1}\left(t_{1}\right)}{\left|\sin \left(\theta_{1}-\theta_{2}\right)\right|}, \\
\left|\theta_{1}-\theta_{2}\right| & \leqq \frac{\pi}{2}-\delta .
\end{aligned}
$$

If the difference $\left(\theta_{E+h}-\theta_{E}\right)(0)$ is close to $\frac{\pi}{2}$, then the above given discussions are not true since $\cos \left(\theta_{E+h}-\theta_{E}\right)(s)$ is close to 0 for the small s. But if $E>\|F\|+1=2$ then

$$
\left|\left(\theta_{E+h}-\theta_{E}\right)\left(t_{0}\right)-\frac{\pi}{2}\right| \geqq \delta_{2}=\delta_{2}\left(t_{0}\right)
$$

(after some time $t_{0}$ ). Applying the Chapman-Kolmogorov equation

$$
p\left(2 t_{0}, \ldots, \ldots\right)=\int p\left(t_{0}, \ldots, \ldots\right) p\left(t_{0} \ldots\right) \ldots
$$

and the estimate $\beta^{\prime \prime}$ ) we achieve $\beta^{\prime}$ ).

Remark. If $\left(\theta_{E+h}-\theta_{E}\right)(0) \approx \frac{\pi}{2}$ and $1+F\left(x_{s}\right)-E \approx 0, s \leqq t_{0}$, then $\left(\theta_{E+h}-\theta_{E}\right)(s)$ may be close to $\pi / 2$ for all $s \leqq t_{0}$ (with the small probability). For this case the following estimate may be established:

$$
\begin{aligned}
& P_{E+h, E}\left(t_{0},\left(x, \theta_{1}, \theta_{2}\right),\left(x^{\prime}, \theta_{1}^{\prime}, \theta_{2}^{\prime}\right)\right) \\
& \quad \leqq C_{2}\left(t_{0}\right) / \max \left(h, \ln 1 / n \cdot\left|\sin \left(\theta_{1}-\theta_{2}\right)\right|\right) .
\end{aligned}
$$

This estimate is sufficient for the future discussions. For the sake of shortness we shall suppose that $E>2$. 
Let us pass on to the proof of $\gamma$ ). We fix $\varepsilon>0$ and consider the following two cases (see [7, Lemma 2]):

a) If $t \leqq h^{-\varepsilon}$ and $\theta_{E+h}(0)=\theta_{E}(0)=\theta$, then for some $C_{0}$

$$
\begin{aligned}
& \mathbf{P}_{(x, \theta, \theta)}\left\{\sin 2\left(\theta_{E+h}-\theta_{E}\right) t(<0\}\right. \\
& \leqq \sum_{k=1}^{\left[t / C_{0}\right]+1} \mathbf{P}_{(x, \theta, \theta)}\left\{\sin \left(\theta_{E+h}-\theta_{E}\right)\left(k C_{0}\right)>\frac{1}{2}\right\} \\
& \leqq\left[\frac{t}{C_{0}}\right] \frac{M_{(x, \theta, \theta)}\left|\sin \left(\theta_{E+h}-\theta_{E}\right)\left(k C_{0}\right)\right|}{C_{1}\left(\varepsilon_{1}\right)} \\
& \leqq C_{2} h^{-\varepsilon} h^{1-\varepsilon_{1}} \leqq C_{3} h^{1-\varepsilon_{2}} .
\end{aligned}
$$

We have used Čebyšev's inequality and the estimate $\alpha$ ). We also have to remember that the phases and their differences can not change too quickly.

b) Let $t>h^{-\varepsilon}$. We put $t^{\prime}=t-h^{-\varepsilon} / 2$ and use the obvious modification of the formula $\left(25^{\prime}\right)$ :

$$
\begin{aligned}
w_{h}(t) & =w_{h}\left(t^{\prime}\right) \exp \left\{-\int_{t^{\prime}}^{t} \ldots\right\}+\int_{t^{\prime}}^{t} \exp \left\{-\int_{u}^{t} \ldots\right\} \ldots \mathrm{du} \\
& =w_{h}\left(t^{\prime}\right) \xi_{1}(t)+\xi_{2}(t) .
\end{aligned}
$$

According to $\alpha)$

$$
\mathbf{P}_{(x, \theta, \theta)}\left\{\left|w_{h}\left(t^{\prime}\right)\right| \geqq \frac{1}{h^{1-\varepsilon}}\right\} \leqq C h^{1-\varepsilon_{1}}
$$

so that

$$
\begin{aligned}
\mathbf{P}_{(x, \theta, \theta)}\left\{\sin 2\left(\theta_{E+h}-\theta_{E}\right)(t)<0\right\} & \leqq C h^{1-\varepsilon_{1}}+M_{(x, \theta, \theta)} \mathbf{P}\left(x_{t^{\prime}}, \theta_{E+h}\left(t^{\prime}\right), \theta_{E}\left(t^{\prime}\right)\right)\left(A_{h}\right), \\
A_{h} & =\left\{\sin 2\left(\theta_{E+h}-\theta_{E}\right)(t)<0, \Omega_{h}\right\},
\end{aligned}
$$

where

$$
\begin{aligned}
& \Omega=\left\{\omega:\left|w_{h}\left(t^{\prime}\right)\right| \leqq \frac{1}{h^{1-\varepsilon}}\right\}, \\
& \mathbf{P}\left\{\Omega_{\hat{h}}\right\} \geqq 1-C h^{1-\varepsilon_{1}}
\end{aligned}
$$

But by Fürstenberg theorem (see [7, Corollary 1 and Lemma 2$]) \exists\left(\delta, \delta_{1}>0\right)$ such that

$$
\mathbf{P}_{(x, \theta, \theta)}\left\{\xi_{1}(t)\right\}>\exp \left\{-\left(t-t^{\prime}\right) \delta\right\} \leqq \exp \left\{-\delta_{1}\left(t-t^{\prime}\right)\right\} \leqq \exp \left\{-\delta_{2} h^{-\varepsilon}\right\}
$$

Since

$$
\begin{aligned}
& \left|\sin \left(\theta_{E+h}-\theta_{E}\right)\left(t^{\prime}\right)\right| \leqq h^{\varepsilon}, \\
& \left|\cos \left(\theta_{E+h}-\theta_{E}\right)\left(t^{\prime}\right)\right| \geqq 1 / 2
\end{aligned}
$$

on $\Omega_{h}$ and $M \xi_{2}^{1-\varepsilon_{2}}(t) \leqq C\left(\varepsilon_{2}\right)$ then repeating the discussions of a) we obtain that

$$
\mathbf{P}\left\{\left|\cos \left(\theta_{E+h}-\theta_{E}\right)(s)\right| \geqq 1 / 4, s \in\left(t^{\prime}, t\right) ; \Omega_{h}\right\} \geqq 1-h^{1-\varepsilon_{3}} .
$$


The last expression means that

$$
\begin{aligned}
& \mathbf{P}_{(x, \theta, \theta)}\left\{\sin 2\left(\theta_{E+h}-\theta_{E}\right)(t)>0, \Omega_{h}\right\} \\
& \quad=\mathbf{P}_{(x, \theta, \theta)}\left\{\operatorname{sgn} \sin \left(\theta_{E+h}-\theta_{E}\right)(t)=\operatorname{sgn} \cos \left(\theta_{E+h}-\theta_{E}\right)(t), \Omega_{h}\right\} \\
& \quad \geqq 1-C h^{1-\varepsilon_{1}}-h^{1-\varepsilon_{3}}-\exp \left\{-\delta_{2} h^{-\varepsilon}\right\} \geqq 1-C_{1} h^{1-\varepsilon_{4}} .
\end{aligned}
$$

From this estimate we obtain $\gamma$ ). The inequality $\gamma^{\prime}$ ) should be established just in the same way.

Now we should prove the important estimate of the transition density

$$
p_{E+h, E}\left(t,(x, \theta, \theta),\left(x^{\prime}, \theta_{1}^{\prime}, \theta_{2}^{\prime}\right)\right) .
$$

Proposition 8. a) If $\left(\theta_{1}^{\prime}-\theta_{2}^{\prime}\right) \bmod \pi \in[C h, \pi-\delta]$ then there exist $t_{0}=t_{0}(C, \delta)$ and $C_{1}=C_{1}(C, \varepsilon, \delta)$ such that for $t \geqq t_{0}$.

$$
p_{E+h, E}\left(t,(x, \theta, \theta),\left(x^{\prime}, \theta_{1}^{\prime}, \theta_{2}^{\prime}\right)\right) \leqq \frac{C_{1} h^{1-\varepsilon}}{\left|\theta_{1}^{\prime}-\theta_{2}^{\prime}\right|^{1-\varepsilon} \max \left(h,\left(\theta_{1}^{\prime}-\theta_{2}^{\prime}\right)\right)}
$$

b) If $\left(\theta_{1}^{\prime}-\theta_{2}^{\prime}\right) \bmod \pi \in[\pi-\delta, \pi]$ then $\exists\left(\alpha, \delta_{1}>0\right)$ such that

$$
p_{E+h, E}\left(t,(x, \theta, \theta),\left(x^{\prime}, \theta_{1}^{\prime}, \theta_{2}^{\prime}\right)\right) \leqq C_{2}\left(\alpha, \delta_{1}\right) h^{\delta_{1}}
$$

for $t>\alpha \ln 1 / h$.

The proof of the case a). By Chapman-Kolmogorov equation

$$
\begin{aligned}
& p_{E+h, E}\left(t,(x, \theta, \theta),\left(x^{\prime}, \theta_{1}^{\prime}, \theta_{2}^{\prime}\right)\right) \\
& =\int p_{E+h, E}\left(t-\tau,\left(x, \theta_{1}, \theta_{2}\right),\left(\tilde{x}, \tilde{\theta}_{1}, \tilde{\theta}_{2}\right)\right) \\
& \quad \cdot p_{E+h, E}\left(\tau,\left(\tilde{x}, \tilde{\theta}_{1}, \tilde{\theta}_{2}\right),\left(x^{\prime}, \theta_{1}^{\prime}, \theta_{2}^{\prime}\right) d \tilde{x} d \tilde{\theta}_{1} d \tilde{\theta}_{2}\right.
\end{aligned}
$$

for $\tau<t$.

Let us note that the formula (25) yields that

hence

$$
\left|\theta_{1}^{1}-\theta_{2}^{\prime}\right| \leqq C(\tau)\left|\tilde{\theta}_{1}-\tilde{\theta}_{2}\right|-C_{1}(\tau) h
$$

$$
\left(\theta_{E+h}(t-\tau)-\theta_{E}(t-\tau)\right) \bmod \pi \in\left(\frac{C}{2} h, \pi-\frac{\delta}{2}\right)
$$

for

$$
\left(\theta_{E+h}-\theta_{E}\right)(t) \bmod \pi \in\left(C h, \pi-\frac{\delta}{2}\right)
$$

and for sufficiently small $\tau=t_{0}$. Using the estimates $\left.\left.\beta^{\prime}\right), \gamma^{\prime}\right)[$ or $\left.\alpha)\right]$ for $\theta_{1}^{\prime}-\theta_{2}^{\prime} \in(0, \delta)$ and the Čebyšev's inequality we obtain (26). The similar discussions are applicable to the case when $\theta_{1}^{\prime}-\theta_{2}^{\prime} \in(\pi-\delta, \pi)$. But in this situation they give us the estimate

$$
p_{E+h, E}\left(t,(x, \theta, \theta),\left(x^{\prime}, \theta_{1}^{\prime}, \theta_{2}^{\prime}\right)\right) \leqq \frac{C_{2} h^{1-\varepsilon}}{\max \left(h, \sin \left(\theta_{1}^{\prime}-\theta_{2}^{\prime}\right)\right)} \leqq C_{2} h^{-\varepsilon}
$$

which is insufficient for our aim. 
To prove b) we should choose the intermediate point $\tau$ depending on $\sin \left(\theta_{1}^{\prime}-\theta_{2}^{\prime}\right)$. If

$$
\tau=\alpha \ln 1 / \max \left(h, \sin \left(\theta_{1}^{\prime}, \theta_{2}^{\prime}\right)\right)
$$

then according to (25) for the sufficiently small $\alpha$

$$
\xi_{2}(t-\tau)=\int_{t-\tau}^{t} \exp \left\{-\int_{n}^{t} \ldots\right\} \cos \left(\theta_{E+h}-\theta_{E}\right)(n) \sin ^{2} \theta_{E+n} d n<0,
$$

if

$$
\left(\theta_{E+h}(t)-\theta_{E}(t)\right) \bmod \pi=\left(\theta_{1}^{\prime}-\theta_{2}^{\prime}\right) \bmod \pi \in(\pi-\delta, \pi)
$$

[since $\cos \left(\theta_{E+h}-\theta_{E}\right)(u)<0$ ]. Moreover, by Fürstenberg theorem (see above) $\exists\left(\delta_{2}, \delta_{3}>0\right)$ :

$$
\left.\mathbf{P}\left\{\xi_{1}=\exp \left\{-\int_{t-\tau}^{t} \ldots d s\right\} \geqq e^{-\delta_{2} t}\right\} \leqq \operatorname{exph}-\delta_{3} \tau\right\} .
$$

But then $\exists\left(\kappa_{1}, \kappa_{2}>0\right)$ :

$$
\begin{gathered}
\mathbf{P}\left\{\left(\theta_{E+h}-\theta_{E}\right)(t-\tau)>\frac{\left(\theta_{E+h}-\theta_{E}\right)(t)}{\left[\max \left(h, \sin \left(\theta_{E+h}-\theta_{E}\right)(t)\right]\right.}\right\} \\
\leqq C \max \left(h, \sin \left(\theta_{1}-\theta_{2}\right)\right)^{\kappa_{2}} .
\end{gathered}
$$

Applying the Chapman-Kolmogorov equation, the estimates $\beta^{\prime}$ ) and (28) (for the sufficiently small $\varepsilon$ ) we have (27). We have proved Proposition 8 and 7 and Theorem 1.

The more attentive analysis of (27) shows that we may put $\delta_{1}=1-\theta$ for every $\theta>0$.

Propositions 7 and 8 gives us the additional information about the structure of the wave functions. We introduce the following functional of the "conductivity type"

$$
\Im_{V}\left(E^{\prime}, E\right)=\frac{1}{V} \sum_{\substack{E_{i}^{(V)<E,} \\ E_{j}^{(V)<E^{\prime}}, E_{i}^{(V) \neq E_{j}^{(V)}}}} \int_{-V}^{V} r_{E_{i}}^{2} r_{E_{j}}^{2}(s) d s .
$$

The formulas (26) and (27) yield

\section{Theorem 2 ("on the repulsion of the wave functions"):}

$$
\Im_{V}\left(E^{\prime}, E\right) \underset{V \rightarrow \infty}{\longrightarrow} \int_{0}^{E^{\prime}} \int_{0}^{E} \varrho\left(e^{\prime}, e\right) d e^{\prime} d e
$$

where

$$
\begin{aligned}
\varrho\left(e^{\prime}, e\right) & =\int_{K \times S^{1} \times S^{1}} \pi_{e^{\prime}, e}\left(x, \theta_{1}, \theta_{2}\right) \pi_{e^{\prime}, e}\left(x, \pi-\theta_{1}, \pi-\theta_{2}\right) d x d \theta_{1} d \theta_{2} \\
& =o\left(\left|e^{\prime}-e\right|^{\varepsilon}\right) .
\end{aligned}
$$

"The density" $\varrho\left(e^{\prime}, e\right)$ characterizes "the mean interaction" between those the wave functions the levels of which are close to $e^{\prime}$ and e resp.

Remark 1. From Theorem 2 it is easy to obtain the following well known fact, namely, for the null temperature and direct current the conductivity $\sigma\left(E_{0}, E_{0}\right)$ of 
the one-dimensional random Markov structure equals zero $\left(E_{0}>0\right.$ is the Fermy energy). Moreover, $\sigma\left(E_{0}, E_{0}+\omega\right)=O\left(\omega^{\varepsilon}\right), \varepsilon>0$. Plainly, the more precise estimates of $\sigma\left(E_{0}, E_{0}+\omega\right)$ can not be achieved when using $\Xi_{V}\left(E^{\prime}, E\right)$. We hope to analyse a conductivity in the our future paper.

Remark 2. The generalization of Theorem 1 for the multidimensional case is the most difficult problem since it is very likely that the wave functions in $R^{v}, v \geqq 3$ are not localized. However the analog of Theorem 1 takes place in some degenerated situations.

\section{References}

1. Zaslavski, G.M. : Preprint IESO-73F, Krasnojarsk 3-41 (1978)

2. Dyson, F.J., Mehta, M.L.: J. Math. Phys. 4, 701-719 (1963)

3. Mehta, M.L.: Random matrices and the statistical theory of energy levels. New York, London: Academic Press 1967

4. Girko, V.L.: Random matrices. Kijev: Naukova Dumka 1979

5. Pokrovskij, V.L.: Letters of GETF 4, 140 (1966)

6. Gol'dseid, I.Ja.: Molčanov, S.A., Pastur, L.A.: Functional Anal. i Prilozen 11, 1-10 (1977); English transl. in Functional Anal. Appl. 11 (1977)

7. Molčanov, S.A.: Izvestija Akad. Nauk SSSR 42, 70-103 (1978); English transl. in Math. USSR Izvestija 12 (1978)

8. Pastur, L.A. : Usp. Mat. Nauk 28 (1973); 1, 3-64 (1969); English transl. in Russian Math. Surveys 28 (1973)

9. Glasman, I.M.: The straight methods of the qualitatıve spectral analysis of the singular and the differential operators. Moskva: Fismatgis 1963

Communicated by Ja. G. Sinai

Received May 5, 1980 